Graphical Abstract
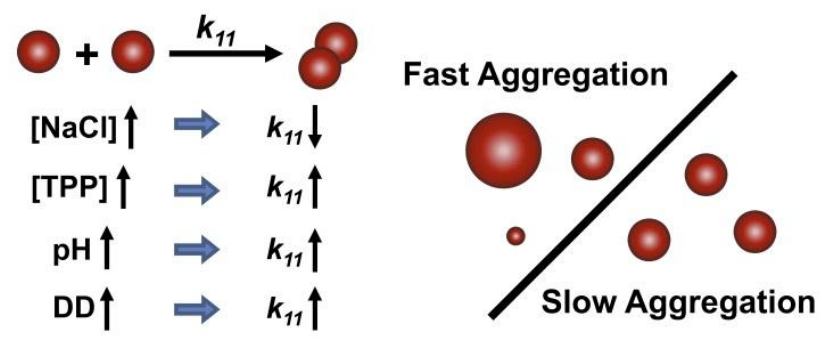


\title{
On the Kinetics of Chitosan/Tripolyphosphate Micro- and Nanogel Aggregation and their Effects on Particle Polydispersity
}

\author{
Yan Huang $^{1, \#}$ and Yakov Lapitsky ${ }^{1,2, *}$ \\ ${ }^{1}$ Department of Chemical and Environmental Engineering, University of Toledo, Toledo, Ohio \\ 43606 \\ ${ }^{2}$ School of Green Chemistry and Engineering, University of Toledo, Toledo, Ohio 43606 \\ ${ }^{\#}$ Current Address: College of Biological Science and Engineering, Fuzhou University, Fuzhou, \\ Fujian, 350108, China \\ *Corresponding Author: yakov.lapitsky@utoledo.edu
}

\begin{abstract}
Submicron chitosan/tripolyphosphate (TPP) particles are widely investigated as nanocarriers for drugs, genes and vaccines. One of the key particle properties that requires control is their size distribution, which depends on the extent of chitosan/TPP primary nanoparticle aggregation into higher-order submicron colloids. To provide a better understanding of this higher-order aggregation process, this study analyzes the factors that control chitosan/TPP particle aggregation kinetics in the presence of free TPP (such as present during particle formation). The aggregation rates exhibit a sharp power-law decrease with the monovalent salt concentration and a power-law increase with the free TPP concentration. Moreover, the aggregation rates increase with the $\mathrm{pH}$ and with the chitosan degree of deacetylation (DD). These variations in aggregation rates reflect the effects of monovalent salt, TPP concentration, $\mathrm{pH}$ and chitosan DD on particle bridging by the surface-bound TPP. Furthermore, these aggregation rates are much faster than those predicted based on Derjaguin and Landau, Verwey and Overbeek (DLVO) interaction potentials, which might reflect nonuniformities in particle shape and charge, and/or complications caused by particle softness. Finally, implications of the above aggregation kinetics on the uniformity of chitosan/TPP micro- and nanogel size are analyzed, where we: (1) show how particle polydispersity can be diminished by lowering the chitosan DD; and (2) explain the opposing results on how chitosan/TPP particle polydispersity is affected by monovalent salt.
\end{abstract}

Keywords: chitosan, tripolyphosphate, microgels, nanoparticles, ionic crosslinking, aggregation, polydispersity 


\section{Introduction}

Submicron particles prepared through the ionic crosslinking of chitosan with tripolyphosphate (TPP) are widely investigated as potential vehicles for drug and gene delivery [1-8]. They form under very mild, aqueous conditions (which help preserve the bioactivity of their payloads) [9], are mucoadhesive $[10,11]$ and can facilitate drug transport across epithelial membranes $[8,11]$. Consequently, they attract special interest for ocular $[4,12]$ and intranasal $[8$, 13] administration of bioactive payloads ranging from small molecules [4, 8, 14], to protein drugs and vaccines $[1,5,6]$, to polynucleotides $[3,15]$.

To ensure their optimal performance, it is vital to control their size distributions. To this end, numerous experimental studies have been performed [2, 14, 16, 17]. These revealed a variety of guidelines (sometimes conflicting ones) on tuning both the particle average size and polydispersity. The average particle size, for instance, has generally been shown to increase with the concentration of the parent chitosan solution and the chitosan molecular weight $[14,17,18]$. Likewise, chitosan/TPP particle size and colloidal stability were demonstrated to be sensitive to the TPP:chitosan ratio $[14,16,17,19]$. When the chitosan monomer (glucosamine) binding sites were in excess, the particle sizes typically decreased with the TPP:glucosamine molar ratio, due to an evident decrease in swelling $[16,17,19]$. Conversely, when multivalent TPP was present in excess, the particles aggregated and ultimately precipitated [14, 16, 19].

In addition to controlling their average size, significant attention has been devoted to minimizing chitosan/TPP particle polydispersity [14, 16, 18-21]. Approaches to doing this have included use of parent chitosan molecules with low molecular weights $[14,18]$, preparation of chitosan samples with highly-uniform molecular weights and degrees of deacetylation (DDs) [21], or varying process and formulation parameters such as the chitosan and TPP concentrations 
$[14,18,19]$, preparation temperature [16] and $\mathrm{pH}[14,20]$, and mixing procedures $[16,18]$.

Recently, we and others have shown that (provided that the ionic strength is not high enough to inhibit particle formation) preparation of chitosan/TPP particles in the presence of monovalent salt can drastically reduce particle polydispersity $[19,22]$. Conversely, Bugnicourt et al., who prepared chitosan/TPP particles with narrow size distributions without the use of salt (by using highly-deacetylated chitosan with a narrow molecular weight distribution [21]), have made opposite observations, where the polydispersities were generally higher at elevated ionic strengths [18]. Such conflicting results have also been reported in studies examining variations in average particle size. Despite studying similar TPP:chitosan ratios, for example, some groups reported particle size to increase with the TPP:chitosan ratio [14], others reported the average particle size to decrease with the TPP:chitosan ratio [17, 23], and others yet reported nonmonotonic TPP:chitosan ratio effects on particle size (where a maximum or minimum particle size occurred at intermediate ratios) $[21,22,24]$. Thus, chitosan/TPP micro- and nanogel size is tuned through trial and error, with few reliable guidelines for achieving predictable control, and limited understanding of the underlying molecular and colloidal interactions.

Despite this limited understanding, the dependence of chitosan/TPP particle size on the method by which the chitosan and TPP are mixed $[20,25]$ clearly indicates that particle size distributions are kinetically controlled. To gain further mechanistic insight into their formation kinetics, we have recently shown these particles to form through a two-step mechanism, where primary 20 - $50 \mathrm{~nm}$ nanoparticles form and then aggregate into the larger, higher-order colloids that are obtained at the end of particle formation process [26]. Thus, the ultimate size distributions of chitosan/TPP particles reflect the extent of their secondary/higher-order aggregation [26], the understanding of which could enable better control over particle size. 
The aggregation events that underlie the ultimate chitosan/TPP micro- and nanogel size require the particles to: (1) collide (i.e., establish surface-to-surface contact); and (2) stick together upon colliding [27]. For lyophobic colloids, it is assumed that the particles always stick (i.e., aggregate) upon colliding due to their strong van der Waals attraction, which dominates at short distances and holds the particles together once they overcome the longer-range electrostatic energy barrier [28]. Conversely, contact between lyophilic particles does not always lead to sticking, due to either a much weaker van der Waals attraction [27, 29, 30], or repulsive solvation [31] and/or steric forces [32]. This is exemplified in a study by Wu et al., where poly(N-isopropylacrylamide) (pNIPAm) microgels (in their water-swollen states) behaved thermodynamically as hard spheres and did not stick upon colliding [29, 33]. This lyophilicity was attributed to the similarity in Hamaker constants between the suspending aqueous medium and water-swollen microgel phases (in other words, negligibly weak van der Waals forces) [29].

Recently, we have postulated that similar phenomena might occur in the case of chitosan/TPP particles [26, 27], which are also water-rich [22, 34] and have, at least qualitatively, been reported to become more (rather than less) colloidally stable when monovalent salt is added - i.e., increasing their collision frequency does not increase their aggregation rate $[19,35]$. Even when the van der Waals attraction is weak, however, such lyophilic particles can still flocculate (or aggregate) through bridging and depletion mechanisms [28]. Bridging flocculation occurs when a flocculant (in this case TPP) simultaneously binds to two particles and causes aggregation by "bridging" the particles together. This bridging has been proposed as the dominant aggregation mechanism during the formation of chitosan/TPP microand nanogels $[14,19]$. Still lacking, however, is a quantitative and systematic analysis of the factors that control these aggregation kinetics. Indeed, despite the extensive literature on the 
aggregation rate constants of colloidal materials [36-38], data on the aggregation rate constants of even other types of polymeric micro- and nanogels (e.g., those formed through covalent crosslinking) remains very limited [39].

Here, to gain quantitative insight into how various parameters (i.e., $\mathrm{NaCl}$ concentration, TPP concentration, $\mathrm{pH}$ and DD-values) affect the aggregation rates, the size evolutions of aggregating chitosan/TPP particles are investigated by light scattering. Though chitosan/TPP particles are typically formed using TPP-limited conditions (so that their aggregation stops once the free TPP is depleted), this study investigates chitosan/TPP particle aggregation in an excess of TPP - i.e., by adding TPP to preformed particles with nearly saturated TPP binding sites. This keeps the free TPP concentration roughly constant throughout each aggregation experiment, so that aggregation rate constants can be obtained from the light scattering data. To further explore the aggregation mechanism, these aggregation rate constants are then compared with the particle collision rates predicted based on Fuchs theory and Derjaguin and Landau, Verwey and Overbeek (DLVO) interaction potentials [28, 40, 41]. Finally, the impacts of these aggregation rates on chitosan/TPP particle polydispersity are discussed, where we: (1) demonstrate how particle polydispersity can be lowered by reducing the chitosan DD; and (2) explain the conflicting results on how their polydispersity is affected by monovalent salt.

\section{Materials and Methods}

2.1. Materials. Millipore Direct-Q 3 deionized water $(18.2 \mathrm{M} \Omega \cdot \mathrm{cm})$ was used in all experiments. Chitosan (viscosity-average molecular weight, $M_{v}=80 \mathrm{kDa}[42]$ ), TPP and $\mathrm{NaCl}$ were purchased from Sigma-Aldrich (St. Louis, USA). The chitosan DD was determined to be $91 \%$ by $\mathrm{pH}$ titration, as described previously [43]. $\mathrm{HCl}(6 \mathrm{~N})$ was purchased from Fisher Scientific (Fair 
Lawn, NJ), while the $\mathrm{NaOH}$ was purchased from VWR (West Chester, PA). All materials were used as received.

2.2. Micro- and Nanogel Preparation. The particles were prepared from parent chitosan and TPP solutions at matching ionic strength and $\mathrm{pH}$-levels (adjusted using $\mathrm{NaCl}, \mathrm{HCl}$ and $\mathrm{NaOH}$ ), where the cationic chitosan had $\mathrm{Cl}^{-}$counterions and the anionic TPP had $\mathrm{Na}^{+}$counterions. To ensure that the free TPP concentration stayed essentially constant during the aggregation experiments, the chitosan/TPP particles were first prepared at the 0.20:1 TPP:glucosamine molar ratios (where the internal chitosan binding sites within the particles, which can adsorb TPP from solution, were nearly saturated [19]). These particles were prepared through a dropwise addition of $1 \mathrm{~mL}$ of $0.16 \mathrm{wt} \%(4.4 \mathrm{mM})$ TPP solution to $10 \mathrm{~mL}$ of $0.04 \mathrm{wt} \%$ (2.2 $\mathrm{mM}$ in its glucosamine units) chitosan solution (both at $\mathrm{pH} 4.0$ and $150 \mathrm{mM} \mathrm{NaCl}$ ) while stirring at $800 \mathrm{rpm}$ with a $8 \mathrm{~mm} \times 3$ mm cylindrical stir bar. The resulting dispersions were then left to stir overnight. To quantify their aggregation kinetics at different TPP concentrations, additional $2-9 \mathrm{~mL}$ of $0.16 \mathrm{wt} \%$ TPP solution (also at $\mathrm{pH} 4.0$ and $150 \mathrm{mM} \mathrm{NaCl}$ ) were rapidly added to the particle dispersions to trigger their aggregation. The mixtures were then stirred for $5 \mathrm{~s}$ to ensure thorough mixing and transferred to dynamic light scattering (DLS) sample cells to monitor changes in their average particle size.

When the parent chitosan and TPP solutions contained $\mathrm{NaCl}$, the resulting particle size depended on the $\mathrm{NaCl}$ concentration. This size variability made the aggregation rates at different $\mathrm{NaCl}$ concentrations difficult to directly compare. To avoid this problem, all particles were first prepared at the same ionic strength by adding $1 \mathrm{~mL}$ of $0.32 \mathrm{wt} \%$ TPP solution to $10 \mathrm{~mL}$ of 0.08 wt\% chitosan (at $\mathrm{pH} 4.0$ with $150 \mathrm{mM} \mathrm{NaCl}$ ) and stirring the mixture overnight. Five $\mathrm{mL}$ of the 
resulting dispersion were then diluted with $5 \mathrm{~mL}$ of $0,50,100,150$ or $200 \mathrm{mM} \mathrm{NaCl}$ solution (also at $\mathrm{pH} 4.0$ ) to achieve a final chitosan concentration of $0.036 \mathrm{wt} \%$ and final $\mathrm{NaCl}$ concentrations of $75,100,125,150$ or $175 \mathrm{mM}$. Ten min after dilution, the final dispersions were tested by DLS to confirm that the particle size distributions did not change. Then, 3-mL aliquots of $0.16 \mathrm{wt} \% \mathrm{TPP}$ solution at corresponding $\mathrm{NaCl}$ concentrations were rapidly added to the particle dispersions to trigger aggregation. The samples were stirred for $5 \mathrm{~s}$ and immediately transferred to a DLS sample cell for the size evolution measurements.

Similarly, to test the $\mathrm{pH}$-effects on the coagulation kinetics, the $\mathrm{pH}$-values of preformed particle dispersions (prepared as described in the previous paragraph at $\mathrm{pH} 4.0$ and $150 \mathrm{mM}$ $\mathrm{NaCl}$ ) were carefully adjusted to $3.50,3.75,4.00,4.25$ or 4.50 (using $0.1 \mathrm{M} \mathrm{HCl}$ and $\mathrm{NaOH}$ solutions) and equilibrated for $10 \mathrm{~min}$. The samples were then probed by DLS to ensure the particle size distribution did not change during the $\mathrm{pH}$ adjustment. Then, $3 \mathrm{~mL}$ of $0.16 \mathrm{wt} \%$ TPP solution (at a matching $\mathrm{pH}$ ) was rapidly added to each dispersion and stirred for $5 \mathrm{~s}$ before monitoring the dispersion for aggregation by DLS.

To investigate the DD effect on the aggregation kinetics, chitosan/TPP particles were prepared using chitosan with 72, 82, 91 and $95 \%$ DD-values. Here, the chitosans with 72, 82 and 95\% DD-values (and fairly similar $M_{v}$-values of $76 \mathrm{kDa}, 73 \mathrm{kDa}$ and $57 \mathrm{kDa}$, respectively) were prepared as described previously [42], using standard deacetylation and reacetylation procedures. Particles from chitosan with 72, 82 and 91\% DD-values were formed by adding 4.0, 3.6 or 3.2 $\mathrm{mL}$ of $0.1 \mathrm{wt} \%$ TPP $(2.75 \mathrm{mM})$ to $10 \mathrm{~mL}$ of $0.1 \mathrm{wt} \%$ chitosan solution (both at $\mathrm{pH} 4.0$ ) while stirring at $800 \mathrm{rpm}$, so that each final mixture was at a 0.2:1 TPP:glucosamine molar ratio. Each dispersion was then stirred overnight, whereupon $4 \mathrm{~mL}$ of each sample were diluted by $6 \mathrm{~mL}$ of $41.7 \mathrm{mM} \mathrm{NaCl}$ solution (pH 4.0) to achieve a final $\mathrm{NaCl}$ concentration of $25 \mathrm{mM}$. 
Conversely, to ensure similar particle size and low polydispersity, particles prepared from 95\% DD chitosan were formed by adding $4.2 \mathrm{~mL}$ of $0.04 \mathrm{wt} \%$ TPP $(1.1 \mathrm{mM})$ to $10 \mathrm{~mL}$ of 0.04 wt $\%$ chitosan solution (both at $\mathrm{pH} 4.0$ and $25 \mathrm{mM} \mathrm{NaCl}$ ) while stirring at $800 \mathrm{rpm}$. The resulting dispersion was stirred overnight, whereupon $10 \mathrm{~mL}$ of the mixture (which were to be used for the aggregation kinetics measurements) were moved into a new $20 \mathrm{~mL}$ scintillation vial. The final particles obtained using the four different DD-values all had similar z-average diameters (of roughly $130 \mathrm{~nm}$, as determined by DLS) and were all at the 0.2:1 TPP:glucosamine ratio. To trigger their aggregation, either 0.15 or $1.5 \mathrm{~mL}$ of $0.16 \mathrm{wt} \%$ TPP solution $(4.4 \mathrm{mM}$; at $\mathrm{pH} 4.0$ and $25 \mathrm{mM} \mathrm{NaCl}$ ) were added to the $10 \mathrm{~mL}$ chitosan/TPP dispersion samples. Each sample was stirred for $5 \mathrm{~s}$ and immediately transferred to a DLS sample cell to monitor its size evolution.

2.3. Light Scattering Analyses. Analyses of chitosan/TPP micro- and nanogel size and aggregation kinetics were primarily performed via DLS, using a Zetasizer Nano ZS dynamic and electrophoretic instrument (Malvern, UK), at $\lambda=633 \mathrm{~nm}$ and a scattering angle of $173^{\circ}$. The initial particle sizes and polydispersity indices (PDIs) were determined via the cumulant analysis as described previously [19]. Conversely, chitosan/TPP particle aggregation kinetics were quantified by tracking the temporal hydrodynamic size change at the onset of their aggregation. Once excess TPP was added to trigger particle aggregation (see Section 3.2), their z-average hydrodynamic radii (obtained through the cumulant analysis of DLS data) were tracked until they increased by $25 \%$. As shown in Fig. 1 , the increase in their normalized radii, $r_{t} / r_{0}$ (where $r_{t}$ is the radius at time $t$ and $r_{0}$ is the initial radius) in these initial aggregation stages was linear. The slopes obtained from the $r_{t} / r_{0}$ versus $t$ plots were then used to obtain the aggregation rate constant $k_{11}$, defined based on Fuchs' expression [44]: 
$\frac{d N_{1}}{d t}=-k_{11} N_{1}^{2}$

where $N_{l}$ was the concentration of unaggregated (singlet) particles. This $k_{11}$ was found by assuming that: (1) the only aggregation occurring at short times is that of singlets into doublets; and (2) that the scattering from the particles obeys the Rayleigh-Gans-Debye (RGD) scattering theory [37]. This assumption was made based on the criterion that $4 \pi r|m-1| / \lambda<<$ (where $m$ is the refractive index ratio between the particle and the medium) [37] - i.e., based on the refractive index of chitosan/TPP particles that was estimated from their chitosan content (around 1.37, via the analysis in Section 3.5), $4 \pi r|m-1| / \lambda \sim 0.01-0.1$. Accordingly, $k_{11}$ was obtained by solving the following equation $[37,45]$ :

$\frac{1}{r_{0}}\left(\frac{d r_{t}}{d t}\right)_{t \rightarrow 0}=\left(\frac{\sin \left(2 r_{0} q\right)}{2 r_{0} q}+1\right) k_{11} N_{0}\left(1-\frac{r_{0}}{r_{h, 2}}\right)$

where $\frac{1}{r_{0}}\left(\frac{d r_{t}}{d t}\right)_{t \rightarrow 0}$ is the normalized rate of hydrodynamic radius change (which equals to the slope of the normalized size evolution curve), $N_{0}$ is the initial particle concentration, $r_{0} / r_{h, 2}$ is the ratio of hydrodynamic radii between the singlets and doublets (which equals 0.725 , assuming that the particles do not coalesce) [37], and $q$ is the light scattering vector. Moreover, $q$ is calculated as:

$q=\left(\frac{4 \pi n_{0}}{\lambda}\right) \sin \left(\frac{\theta}{2}\right)$

where $\lambda$ is the wavelength of the light source, $n_{0}$ is the refractive index of the suspending medium, $\theta$ is the angle between incident light and the detector. 


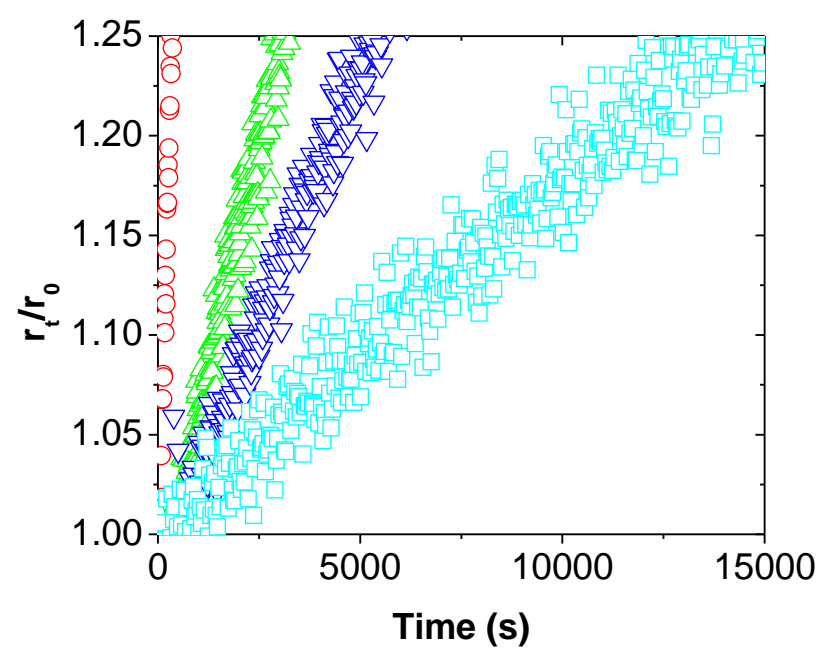

Fig. 1. Representative DLS data showing the normalized size evolutions of chitosan/TPP particles (prepared from 91\% DD chitosan at a 0.2:1 TPP:glucosamine molar ratio) and (O) 100 $\mathrm{mM},(\triangle) 125 \mathrm{mM},(\nabla) 150 \mathrm{mM}$ and $(\square) 175 \mathrm{mM} \mathrm{NaCl}$. These data sets were collected at $\mathrm{pH}$ 4.0 and in the presence of $1.0 \mathrm{mM}$ excess TPP $(n=3)$.

To confirm the validity of the $k_{11}$-values obtained using the RGD theory-based DLS analysis, $k_{11}$-values were also calculated using a combined DLS + static light scattering (SLS) analysis, which relaxes the RGD scattering assumption. These calculations were made based on changes in both the particle radius and average (static) light scattering intensity, $I(q, t)$ as $[37,45]$ :

$$
\frac{1}{r_{0}}\left(\frac{d r_{t}}{d t}\right)_{t \rightarrow 0}=\left[\frac{1}{I(q, 0)}\left(\frac{d I(q, t)}{d t}\right)_{t \rightarrow 0}+k_{11} N_{0}\right]\left(1-\frac{r_{0}}{r_{h, 2}}\right)
$$

where $I(q, 0)$ is the initial light scattering intensity from the dispersion and $I(q, t)$ is the light scattering intensity at time $t$. This DLS + SLS analysis yielded very similar $k_{11}$-values to those obtained using the RGD theory-based DLS analysis (Supporting Information, Fig. S1) and validated the DLS-based $k_{11}$-estimates. Consequently, and because data collected by the DLS + SLS method was less precise (i.e., $d I(q, t) / d t$ at the $173^{\circ}$ scattering angle was so small that its goodness of fit was limited by the scatter in the $I(q, t)$ data), the remainder of this paper focuses 
solely on the DLS-based $k_{11}$-values. Each light scattering measurement was reproduced three times to obtain average values and standard deviations.

\begin{abstract}
2.4. Electrophoretic Light Scattering. The apparent $\zeta$-potentials of the micro- and nanogels were obtained via electrophoretic light scattering. This was achieved using the Zetasizer Nano ZS instrument (Malvern, UK), where the $\zeta$-potentials were estimated from the electrophoretic mobility using the Smoluchowski model [46]. These measurements were performed on all chitosan/TPP dispersions used in the aggregation rate tests, which were analyzed immediately after excess TPP addition. Each $\zeta$-potential measurement was repeated thrice.
\end{abstract}

2.5. Capillary Viscometry. To determine $N_{0}$, the relative viscosity, $\eta_{r}$, was determined using a Cannon-Ubbelohde dilution capillary viscometer (Size 75; Cannon Instrument Co., State College, PA). This was achieved by dividing the particle dispersion viscosity, $\eta$, by the solvent viscosity, $\eta_{s}$, from which the particle volume fraction, $\phi$, was estimated by Einstein's theory, which assumes the particles to be rigid spheres [28]:

$\eta_{r}=\frac{\eta}{\eta_{s}}=1+2.5 \phi+k_{1} \phi^{2}+\ldots$

Because the volume fraction of chitosan/TPP microgels was only $1-2 \%$, the higher-order terms in Eqn. 5 (e.g., $k_{1} \phi^{2}$ ) were negligible. The $N_{0}$ was therefore calculated by dividing $\phi$ by the volume of a single particle:

$$
N_{0}=\frac{\phi}{\frac{4}{3} \pi r_{0}^{3}}
$$


This estimation was reasonable because the particle PDI-values were low (below 0.13 for all tests used for the $N_{0}$ quantification), which ensured reliable $r_{0}$-values for the number concentration calculation. To ensure reproducibility, each viscosity measurement was repeated thrice.

\section{Results and Discussion}

3.1. Ionic Strength Effect. The first effect examined was that of ionic strength (i.e., the $\mathrm{NaCl}$ concentration effect). Similar to the particle formation rates reported by us earlier [26], chitosan/TPP particle aggregation rates diminished sharply with the $\mathrm{NaCl}$ concentration (see Fig. 1). When the $\mathrm{NaCl}$ concentration was $100 \mathrm{mM}$, the normalized hydrodynamic particle radius $\left(r_{0} / r_{t}\right)$ increased to 1.25 within $10 \mathrm{~min}$. When the $\mathrm{NaCl}$ concentration was gradually increased to $175 \mathrm{mM}$, however, the time required for the same size increase was extended to roughly $4 \mathrm{~h}$. This enhanced colloidal stability at higher monovalent salt concentrations further confirmed our previous findings, where higher excess TPP concentrations were required to aggregate chitosan/TPP microgels in the presence of $150 \mathrm{mM} \mathrm{NaCl}$ than in the absence of added $\mathrm{NaCl}$ [19].

After fitting these curves to Eqn. 2 (in Section 2.3), the $k_{11} N_{0}$-values at each $\mathrm{NaCl}$ concentration were obtained (Table 1). The $N_{0}$-value was then determined via capillary viscometry to calculate $k_{11}$. Notably, the micro- and nanogel volume fraction determined by this analysis also enabled the determination of the chitosan content within the particles (i.e., by dividing the overall chitosan concentration by $\phi$ [47]). Thus, based on a 0.073 wt $\%$ overall chitosan concentration and a $\phi$ of $1.03 \mathrm{vol} \%$ (measured in $150 \mathrm{mM} \mathrm{NaCl}$ at $\mathrm{pH} 4.0$ ), the particles were estimated to contain roughly $7.1 \mathrm{wt} \%$ chitosan. This very low polymer content was consistent with those reported by other groups [22, 34]. Moreover, it suggested a highly swollen 
micro- and nanogel structure and, consequently, a very weak van der Waals attraction between the micro- and nanogels (vide infra). Likewise, as shown by the virtually constant $r_{0}$ and $\zeta$ potential in Table 1, the polymer content within the particles (which were all prepared in 150 $\mathrm{mM} \mathrm{NaCl}$ ) was largely unaffected by the variations in the ambient $\mathrm{NaCl}$ concentration. This invariance in particle size and composition shows that their swelling remains unchanged and confirms that the only significant change in the chitosan/TPP dispersions was their ionic strength.

Table 1. Experimental parameters and fitted $k_{11}$-values showing the $\mathrm{NaCl}$ effect on the chitosan/TPP micro- and nanogel aggregation rates at $\mathrm{pH} 4.0$ and in the presence of $1.0 \mathrm{mM}$ excess TPP (mean \pm standard deviation).

\begin{tabular}{ccccccc}
\hline $\begin{array}{c}{[\mathrm{NaCl}]} \\
(\mathrm{mM})\end{array}$ & $\begin{array}{c}r_{0} \\
(\mathrm{~nm})\end{array}$ & $\begin{array}{c}\zeta \\
(\mathrm{mV})\end{array}$ & $\begin{array}{c}d\left(r_{t} / r_{0}\right) / d t \\
(1 / \mathrm{s})\end{array}$ & $\begin{array}{c}k_{l 1} N_{0} \\
(1 / \mathrm{s})\end{array}$ & $\begin{array}{c}N_{0} \\
\left(1 / \mathrm{m}^{3}\right)\end{array}$ & $\begin{array}{c}k_{11} \\
\left(\mathrm{~m}^{3} / \mathrm{s}\right)\end{array}$ \\
\hline 75 & $79.8 \pm 1.1$ & $21.2 \pm 0.8$ & $(5.7 \pm 0.9) \times 10^{-3}$ & $(2.6 \pm 0.4) \times 10^{-2}$ & $(1.8 \pm 0.1) \times 10^{18}$ & $(1.5 \pm 0.2) \times 10^{-20}$ \\
100 & $73.0 \pm 1.6$ & $21.4 \pm 2.9$ & $(8.2 \pm 1.6) \times 10^{-4}$ & $(3.6 \pm 0.7) \times 10^{-3}$ & $(1.8 \pm 0.1) \times 10^{18}$ & $(2.0 \pm 0.4) \times 10^{-21}$ \\
125 & $81.3 \pm 1.1$ & $21.6 \pm 1.1$ & $(9.7 \pm 1.4) \times 10^{-5}$ & $(4.5 \pm 0.6) \times 10^{-4}$ & $(1.8 \pm 0.1) \times 10^{18}$ & $(2.5 \pm 0.4) \times 10^{-22}$ \\
150 & $79.5 \pm 0.9$ & $22.0 \pm 0.9$ & $(5.2 \pm 0.5) \times 10^{-5}$ & $(2.4 \pm 0.2) \times 10^{-4}$ & $(1.8 \pm 0.1) \times 10^{18}$ & $(1.3 \pm 0.1) \times 10^{-22}$ \\
175 & $82.1 \pm 0.5$ & $22.3 \pm 0.7$ & $(1.8 \pm 0.3) \times 10^{-5}$ & $(8.2 \pm 1.2) \times 10^{-5}$ & $(1.8 \pm 0.1) \times 10^{18}$ & $(4.6 \pm 0.7) \times 10^{-23}$ \\
\hline
\end{tabular}

As indicated in Table 1, the aggregation rate constant $k_{11}$ (obtained via Eqn. 9) decreased drastically from $1.5 \times 10^{-20}$ to $4.6 \times 10^{-23} \mathrm{~m}^{3} / \mathrm{s}$ as the $\mathrm{NaCl}$ concentration increased from $75 \mathrm{mM}$ to $175 \mathrm{mM}$. Plotting $k_{11}$ against $\mathrm{NaCl}$ concentration (Fig. 2) revealed $k_{11}$ to scale approximately with $[\mathrm{NaCl}]^{-6.8}$. This strong dependence is qualitatively similar to that of chitosan/TPP microand nanogel formation rates [26], which (based on the characteristic times of the increase in light scattering intensity during their formation) were estimated to scale with $[\mathrm{NaCl}]^{-5.0}$. 


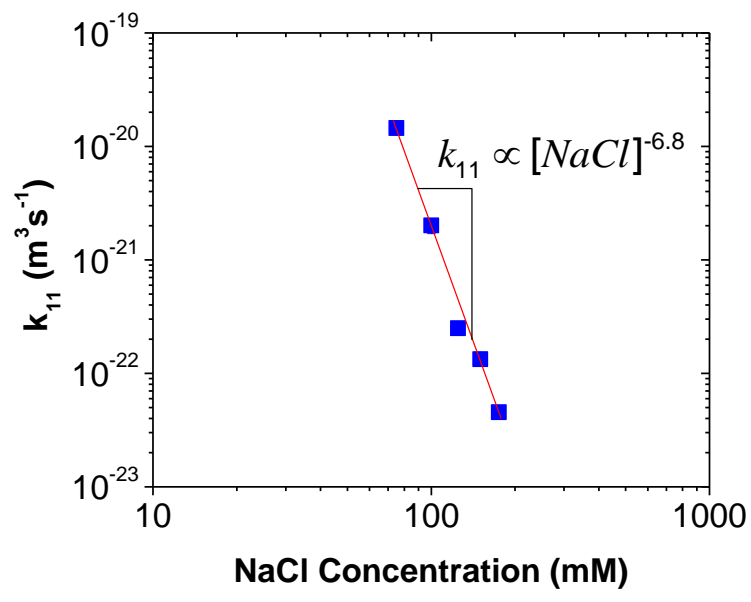

Fig. 2. $\mathrm{NaCl}$ effect on the $k_{11}$-values obtained at $\mathrm{pH} 4.0$ and in the presence of $1.0 \mathrm{mM}$ excess TPP for particles prepared from 91\% DD chitosan (the solid line shows the power law fit and the error bars are standard deviations; $n=3$ ). Because the error bars are obscured by the symbols, the standard deviations are also provided in Table 1.

Though NaCl-enhanced colloidal stability has previously been reported (albeit by morequalitative measurements) by both us and others [19, 35], different explanations for this phenomenon have been proposed. One interpretation was that higher $\mathrm{NaCl}$ concentrations frustrate the binding of TPP ions to the chitosan/TPP particle surfaces and inhibit their bridging flocculation (which was argued to be the primary mechanism by which these lyophilic particles aggregate) [19, 27]. Another interpretation, however, attributed the high colloidal stability at elevated ionic strengths to the smaller size and higher compactness of chitosan/TPP micro- and nanogels, which was speculated to cause fewer collisions and slower chain entanglement between the colliding particles [35]. Specifically, this second interpretation argued that the smaller particle size (which prevented sedimentation-induced collisions) and slower chain entanglement counterbalanced the lower electrostatic repulsive potentials at elevated ionic strengths and led to slower aggregation [35]. This view, however, is opposed by the data in Table 1, which shows that the aggregation kinetics vary significantly even when the size, compactness 
and $\zeta$-potential remain virtually unchanged. Based on this, and the well-characterized weakening of chitosan/TPP binding in the presence of $\mathrm{NaCl}[19,42]$, we confirm that the enhanced colloidal stability reflects a reduction in TPP bridging.

3.2. TPP Concentration Effect. Similar measurements were also performed to investigate the effect of TPP concentration on the aggregation kinetics. Here, the $\mathrm{pH}$ and $\mathrm{NaCl}$ concentration were fixed at 4.0 and $150 \mathrm{mM}$ respectively, while varying the excess (i.e., free) TPP concentration from 0.68 to $1.98 \mathrm{mM}$. As shown in Fig. $3, k_{11}$ increased drastically with the TPP concentration and scaled approximately with $[\mathrm{TPP}]^{4.9}$, which was identical to the scaling of the chitosan/TPP particle formation kinetics reported earlier [26]. The particle sizes and $\zeta$-potentials were also essentially independent of the TPP concentration (see Supporting Information, Table S1) which, again, eliminated any possible differences in particle compactness and sedimentation properties [35]. Likewise, because the variations in the free TPP concentrations used in this experiment had minimal impact on the Debye screening length, $\kappa^{-1} \approx 1 \mathrm{~nm}$ (i.e., the concentration of free TPP was very low relative to that of $\mathrm{NaCl}$ ) [28], the electrostatic repulsion between the particles was hypothesized to remain virtually the same. Accordingly, the strong impact of TPP concentration on the aggregation kinetics again indicated chitosan/TPP micro- and nanogel aggregation to mainly reflect differences in TPP bridging (which can be strongly affected by the competitive binding of TPP and $\mathrm{Cl}^{-}$ions to the particle surface). The similar $\mathrm{NaCl}$ and TPP concentration effects on the micro- and nanogel formation and aggregation processes might stem from their similar mechanisms; micro- and nanogel formation occurs through aggregation of primary nanoparticles [26], while the agglomeration studied here is the higher-order aggregation of larger, secondary aggregates. Moreover, the similar scaling of these rates with the free TPP 
concentration suggests that the aggregation of primary particles, similar to the higher-order aggregation explored here, requires their bridging by TPP.

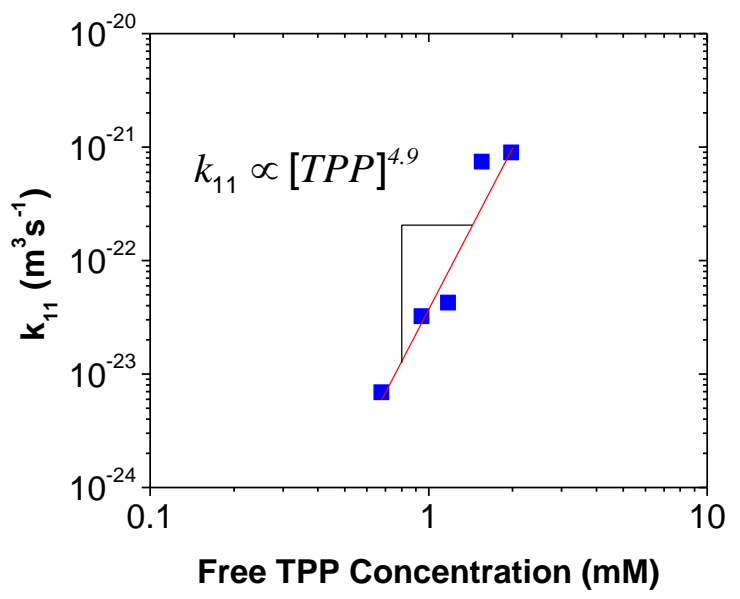

Fig. 3. Free TPP concentration effect on the $k_{11}$-values obtained at $\mathrm{pH} 4.0$ and in the presence of $150 \mathrm{mM} \mathrm{NaCl}$ for particles prepared from 91\% DD chitosan (the solid line is the power law fit and the error bars are standard deviations; $n=3$ ). Because the error bars are obscured by the symbols, the standard deviations are also provided in Table $\mathrm{S} 1$.

3.3. $p H$ Effect. To investigate the $\mathrm{pH}$ effect on chitosan/TPP micro- and nanogel aggregation, preformed particles were aggregated at varying $\mathrm{pH}$-values and at $\mathrm{NaCl}$ and excess TPP concentrations fixed at $150 \mathrm{mM}$ and $0.94 \mathrm{mM}$, respectively. As indicated in Fig. $4 \mathrm{a}, k_{11}$ diminished from $9.3 \times 10^{-22}$ to $3.1 \times 10^{-24} \mathrm{~m}^{3} / \mathrm{s}$ as the $\mathrm{H}^{+}$concentration increased from $3.2 \times 10^{-5}$ to $3.2 \times 10^{-4} \mathrm{M}$ (i.e., as the $\mathrm{pH}$ was reduced from 4.5 to 3.5 ), where $k_{11}$ did not follow a simple power law scaling with $\left[\mathrm{H}^{+}\right]$. Since the effective $p K_{a}$ of chitosan is around $6.0-6.5[48,49]$, the protonation of the chitosan amine groups should be nearly-full and remain virtually-unchanged between $\mathrm{pH} 3.5$ and 4.5. Conversely, TPP (which is a polyprotic acid with $p K_{a, 3}=2.3$ and $p K_{a, 4}$ $=6.3[50])$ changes ionization states within that $\mathrm{pH}$-range. Thus, the change in aggregation kinetics likely reflects the protonation (and charge reduction) on the TPP at lower pH-values, which results in its weaker binding and diminished bridging capability. 
(a)

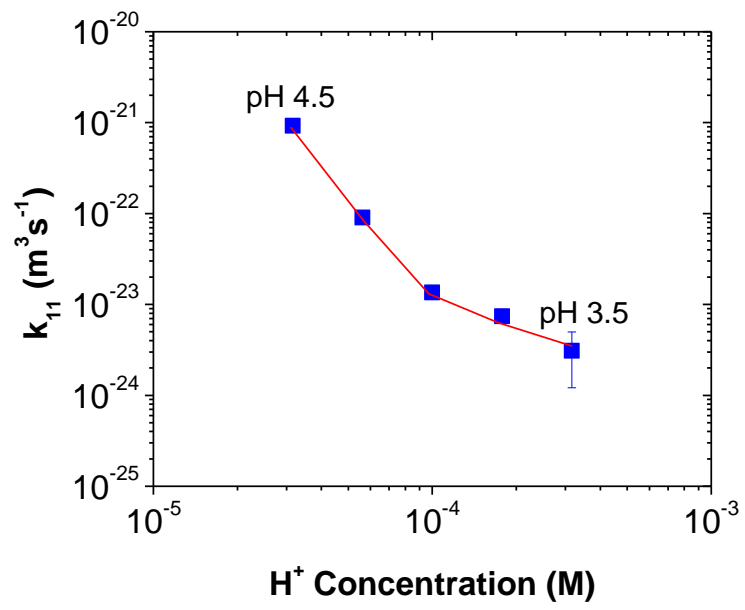

(b)

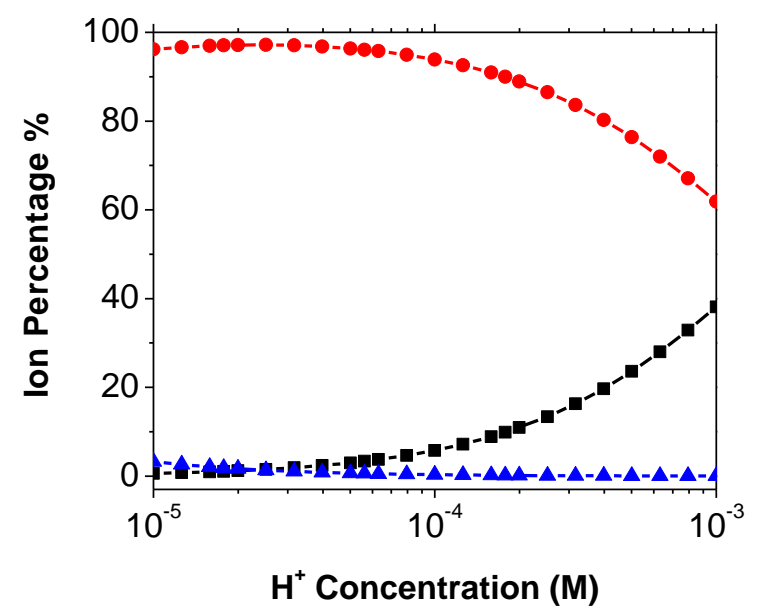

Fig. 4. The effect of $\mathrm{H}^{+}$concentration on (a) the $k_{11}$-values (the solid line is guide to the eyes) obtained in the presence of $150 \mathrm{mM} \mathrm{NaCl}$ and $1.0 \mathrm{mM}$ free TPP (DD = 91\%); and (b) the percentage of (--) $\mathrm{TPP}^{2-},(-\bullet-) \mathrm{TPP}^{3-}$, and $(-\mathbf{\Lambda}-) \mathrm{TPP}^{4-}$ in total TPP estimated from the $p K_{a}$ values of TPP using the Henderson-Hasselbalch equation. The error bars are standard deviations, which are also provided in Table $\mathrm{S} 2(\mathrm{n}=3)$.

Unlike directly changing the TPP concentration, the change in $\mathrm{pH}$ leads to nonlinear changes in the concentrations of free $\mathrm{TPP}^{2-}, \mathrm{TPP}^{3-}$ and $\mathrm{TPP}^{4-}$ ions. This nonlinear change is illustrated in Fig. 4b, which shows how the the percentages TPP ions at each ionization state varied within the range of investigated $\mathrm{pH}$-values (as predicted by the Henderson-Hasselbalch equation). Thus, the more-complicated trend in aggregation kinetics shown in Fig. 4a might reflect these non-linear changes in ionization state. Moreover, the break in the $k_{11}$ versus $\mathrm{H}^{+}$ concentration curve might reflect a switch in the dominant ion type underlying the changes in the aggregation rate - e.g., changing $\mathrm{TPP}^{3-}$ concentrations determining $k_{11}$ at low $\mathrm{pH}$-values (where there is almost no $\mathrm{TPP}^{4-}$ ) and changing $\mathrm{TPP}^{4-}$ concentrations determining $k_{11}$ at higher $\mathrm{pH}$-values (where the $\mathrm{TPP}^{3-}$ concentration remains virtually unchanged). Because the binding of TPP to polycations can affect its $p K_{a}$-values [51], no further quantitative analysis of these effects was performed. 
3.4. DD Effect. To investigate the effect of chitosan DD on the aggregation kinetics, the same experiments were repeated at different $\mathrm{DD}$-values (with the $\mathrm{pH}$ and $\mathrm{NaCl}$ concentration held constant at 4.0 and $25 \mathrm{mM}$, respectively). Fig. 5 shows the $r_{t} / r_{0}$ evolution over time when the excess TPP was added to trigger the aggregation. For particles prepared from $72 \%$ DD chitosan, the aggregation was hardly detected when $0.57 \mathrm{mM}$ excess TPP was added (Fig. 5a). The $r_{t} / r_{0}$ increased slightly from 1.00 to 1.05 upon the addition of $0.57 \mathrm{mM}$ of excess TPP and thereafter remained constant over several hours, thus indicating a very slow aggregation process and a colloidally stable dispersion. When the DD was increased to $82 \%$, however, the aggregation rate increased drastically and the $r_{t} / r_{0}$ rose from 1.00 to roughly 1.30 within 3 min (with macroscopic precipitation occurring within $10 \mathrm{~min})$.

(a)

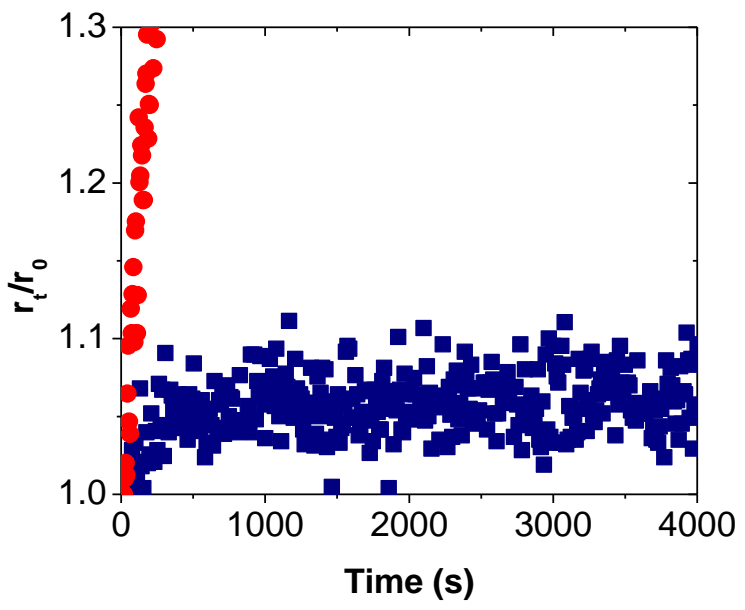

(b)

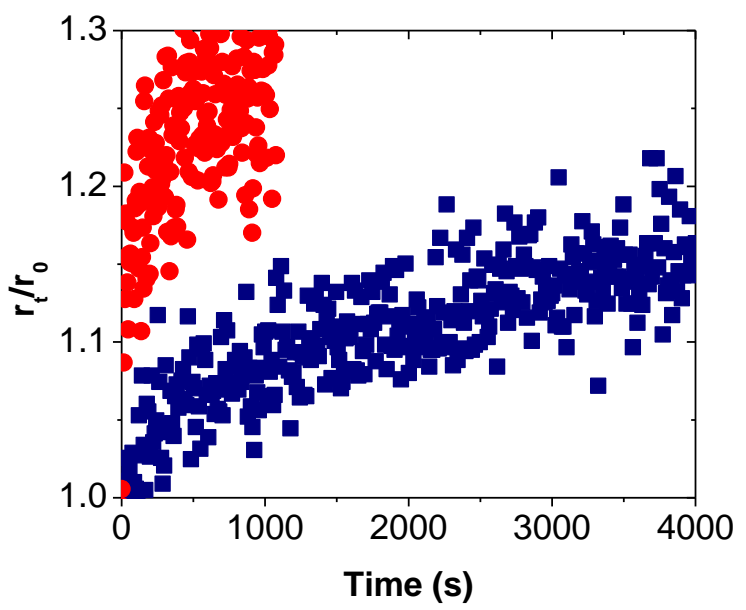

Fig. 5. Representative $r_{t} / r_{0}$ evolutions in the presence of: (a) $0.57 \mathrm{mM}$ excess TPP obtained using

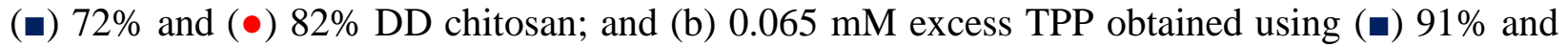
(•) $95 \%$ DD chitosan (each obtained at $\mathrm{pH} 4.0$ and $25 \mathrm{mM} \mathrm{NaCl} ; \mathrm{n}=3$ ).

When the DD was further increased to $91 \%$, the aggregation was far too rapid to be detected using $0.57 \mathrm{mM}$ excess TPP. Thus, the measurements were performed using a much lower excess TPP concentration $(0.065 \mathrm{mM})$. The aggregation of particles with $91 \%$ DD was 
slow but detectable, with $r_{t} / r_{0}$ increasing from 1.00 to 1.15 over roughly $1 \mathrm{~h}$ (see Fig. 5b). The size evolution, however, became rapid again as the DD was raised by just $4 \%$ to $95 \%$, where $r_{t} / r_{0}$ jumped to roughly 1.15 even before the first data point was collected (i.e., within approximately $15 \mathrm{~s})$ and reached 1.25 within $10 \mathrm{~min}$. The burst increase in size during mixing could be explained by non-instantaneous mixing and local aggregation during TPP addition, where (before the TPP solution was mixed with the chitosan/TPP dispersion) the local free TPP concentration was initially much higher than $0.065 \mathrm{mM}$ and resulted in more-rapid aggregation. Alternatively, at the low excess TPP concentration used in this experiment, the consumption of TPP ions by the few free chitosan amine groups might have reduced the free TPP concentration over time and therefore reduced the aggregation rate. Regardless of the reason for the burst increase, Fig. 5 showed that the aggregation kinetics of chitosan/TPP particles increased drastically with the chitosan DD-values, and likely reflected the stronger chitosan/TPP binding (which was recently demonstrated by isothermal titration calorimetry [42]). Thus, in addition to varying with the ionic strength, TPP concentration and $\mathrm{pH}$, the aggregation kinetics can vary with the type of chitosan used, and can be sensitive to even small differences in the DD-values. This is consistent with the recent qualitative findings that the TPP:glucosamine molar ratios where the onset of rapid micro- and nanogel aggregation occurs increases substantially when the DD-value is diminished [42].

3.5. Comparison with Theoretical Predictions. As a first attempt to model chitosan/TPP microand nanogel aggregation, it might be useful to compare the experimental aggregation rates with theoretical predictions based on DLVO potentials, which estimate the interparticle potentials as the sum of van der Waals $\left(\phi_{A}\right)$ and electrostatic $\left(\phi_{E}\right)$ contributions [28, 40, 41]. These potentials 
are derived based on the assumption of uniformly-charged particles with well-defined fluid-solid boundaries. For spherical particles with uniform sizes, $\phi_{A}$ and $\phi_{E}$ are given by [28]:

$$
\begin{aligned}
& \phi_{A}=-\frac{A}{6}\left[\frac{2}{s^{2}+4 s}+\frac{2}{s^{2}+4 s+4}+\ln \left(\frac{s^{2}+4 s}{s^{2}+4 s+4}\right)\right] \\
& \phi_{E}=64 \pi r_{0} k_{B} T n_{\infty} \kappa^{-2} \Upsilon_{0}^{2} \exp \left(-\kappa r_{0} s\right) \\
& \Upsilon_{0}=\frac{\exp \left(e \psi_{0} / 2 k_{B} T\right)-1}{\exp \left(e \psi_{0} / 2 k_{B} T\right)+1}
\end{aligned}
$$

where $A$ is the Hamaker constant, $s$ is the normalized surface-to-surface distance between the particles (given by the distance, $d$, divided by the particle radius, $r_{0}$ ), $k_{B}$ is the Boltzmann constant, $T$ is the absolute temperature, $n_{\infty}$ is the aqueous salt concentration, $\kappa$ is the DebyeHückel parameter, and $\psi_{0}$ is the surface electrostatic potential of the particles, which (due to a lack of structural information about the softness of the particle surface $[52,53])$ was roughly estimated as the apparent $\zeta$-potential. Here [28]:

$\kappa=\left(\frac{2 e^{2} N_{A} I}{\varepsilon_{0} \varepsilon_{r} k_{B} T}\right)^{0.5}$

where $e$ is the charge of an electron, $N_{A}$ is the Avogadro number, $I$ is the ionic strength in the suspending medium, $\varepsilon_{r}$ is the relative dielectric constant of water and $\varepsilon_{0}$ is the dielectric constant of vacuum.

Based on the above potentials, the theoretical collision rate constant between identical particles can be estimated via the modified Fuchs expression as [36, 54]:

$$
\begin{aligned}
& k_{11}=\frac{4 k_{B} T}{3 \eta_{s}}\left[\int_{0}^{\infty} \beta \frac{\exp \left[\left(\phi_{E}+\phi_{A}\right) / k_{B} T\right]}{(2+s)^{2}} d s\right]^{-1} \\
& \beta \approx \frac{6 s^{2}+13 s+2}{6 s^{2}+4 s}
\end{aligned}
$$


which models how the frequency of collisions between smooth, spherical particles is affected by their interaction potentials (e.g., the DLVO potentials) and repulsive hydrodynamic forces, accounted for by $\beta$ [55]. In the case of lyophobic colloids, where surface-to-surface contact between the particles always leads to aggregation, this expression is typically used to quantify the aggregation rates. In the case of the lyophilic chitosan/TPP particles, however (where not all collisions lead to aggregation), this expression only provides a collision rate constant, which will typically be faster than the aggregation rate.

As discussed in Section 3.1, the polymer content in the chitosan/TPP micro- and nanogels used in the $\mathrm{NaCl}$ effect experiments was only $7.1 \mathrm{wt} \%$. Accordingly, the Hamaker constant for the particles in water can be estimated from the refractive indices and dielectric constants of chitosan and water. Ignoring the effect of TPP (which constitutes less than $2 \%$ of the total particle weight), this can be done using an approximation to the Lifshitz theory proposed by Israelachvili $[56,57]$ :

$$
A_{121} \approx \frac{3}{4} k_{B} T\left(\frac{\varepsilon_{1}-\varepsilon_{2}}{\varepsilon_{1}+\varepsilon_{2}}\right)^{2}+\frac{3 h v}{16 \sqrt{2}} \frac{\left(n_{1}^{2}-n_{2}^{2}\right)^{2}}{\left(n_{1}^{2}+n_{2}^{2}\right)^{3 / 2}}
$$

where $\varepsilon_{1}$ and $\varepsilon_{2}$ are the respective dielectric constants for chitosan/TPP particles and water, $h$ is Planck's constant, $n_{1}$ and $n_{2}$ are the refractive indices of chitosan/TPP particles and water, and $v$ is the frequency of light used to measure the refractive indices [57]. The dielectric constant $\varepsilon_{1}$ and refractive index $n_{l}$ of chitosan/TPP micro- and nanogels were estimated using the simple additive relations [57]:

$$
\begin{aligned}
& \varepsilon_{1}=\phi_{c s} \varepsilon_{c s}+\left(1-\phi_{c s}\right) \varepsilon_{\text {water }} \\
& n_{1}=\phi_{c s} n_{c s}+\left(1-\phi_{c s}\right) n_{\text {water }}
\end{aligned}
$$


where $\varepsilon_{c s}$ and $\varepsilon_{w a t e r}$ are the dielectric constants of pure chitosan (7) and water (78), $n_{c s}$ and $n_{\text {water }}$ are the refractive indices of pure chitosan (1.6) and water (1.33) [57], and $\phi_{\text {chitosan }}$ is the volume fraction of chitosan in the particle (roughly approximated as its mass fraction - i.e., $7.1 \%$ ). Combining Eqns. 14 and 15 yielded a Hamaker constant on the order of $10^{-22} \mathrm{~J}$, which (as expected) was very small compared to the typical values of $10^{-20}-10^{-19} \mathrm{~J}$ reported for lyophobic colloids [28]. Using this Hamaker constant, and the experimentally measured particle sizes and $\zeta$-potentials from Table 1, the interparticle interaction potentials were calculated using Eqns. 7 and 8 as $\phi_{A}+\phi_{E}$ (see Fig. 6a). The electrostatic repulsive potential, $\phi_{E}$ (solid line in Fig. 6a), was much higher than the van der Waals attractive potential, $\phi_{A}$ (dashed line in Fig. 6a). This led to a large repulsive barrier (of roughly $35 k_{B} T$ ) in the net interaction potential (dotted line in Fig. 6a) and prevented the particles from colliding.

(a)

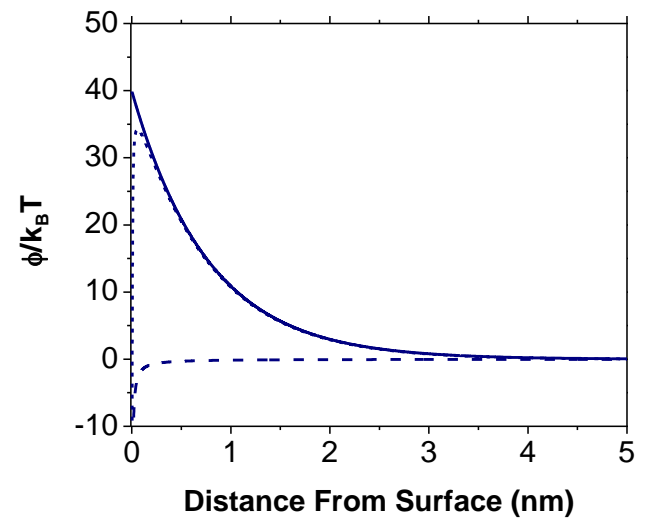

(b)

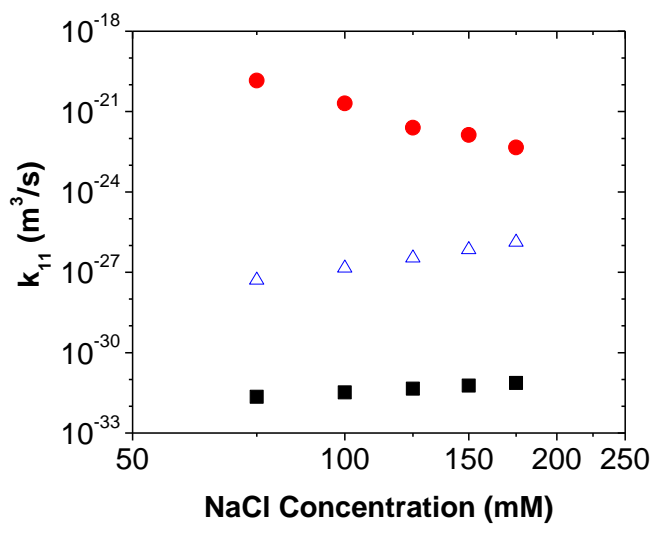

Fig. 6. Plots showing (a) representative particle interaction potentials: (solid line) $\phi_{E}$, (dashed line) $\phi_{A}$ and (dotted line) net potential $\left(\phi_{A}+\phi_{E}\right)$ versus distance from particle surface when $A_{121}=$ $1 \times 10^{-22} \mathrm{~J}, \zeta=22 \mathrm{mV}, r_{0}=80 \mathrm{~nm}$ and $[\mathrm{NaCl}]=150 \mathrm{mM}$; and (b) the aggregation rate constants $(\bullet)$ determined experimentally via DLS (from Table 1) compared to the collision rate constants calculated using Eqn. 10 when (-) $A_{121}=1 \times 10^{-22} \mathrm{~J}$ and $(\Delta) A_{121}=1 \times 10^{-21} \mathrm{~J}$.

This strong repulsive energy barrier gave rise to very slow theoretical collision rates at all examined ionic strengths (solid squares in Fig. 6b). These collision rate constants (calculated 
using Eqn. 10) were around $10^{-32}-10^{-31} \mathrm{~m}^{3} / \mathrm{s}$, and increased slightly with ionic strength due to the reduced electrostatic repulsion between the particles. These theoretical collision rates, however, were much lower than the experimental aggregation rates in Table 1 (circles in Fig. 6b), which ranged between $10^{-20}$ and $10^{-23} \mathrm{~m}^{3} / \mathrm{s}$. Because aggregation events were expected to be less frequent than collisions for chitosan/TPP particles, this discrepancy revealed a gross theoretical underprediction of the collision and aggregation rates. Considering the uncertainty in the estimated $A_{121}$-value, a sensitivity analysis was also performed by increasing $A_{121}$ by one order of magnitude (to $10^{-21} \mathrm{~J}$; see Supplementary Information, Fig. S2a for the interaction potentials) i.e., to probe whether the low theoretical collision rates might reflect an underprediction of $A_{121}$. Even with this enhanced van der Waals attraction, however, the theoretical $k_{11}$-values (open triangles in Fig. 6b) were still much lower than the experimental values. Hence, the discrepancies between the experimental and theoretical $k_{11}$-values were likely not caused by a low $A_{121}$ estimate.

Four other possible explanations emerge for this large deviation between the experimental and theoretical collision rates. The first is that the particles are not perfectly spherical. Because chitosan/TPP micro- and nanogels are aggregates of roughly $30-\mathrm{nm}$ primary nanoparticles [26], they tend to have "raspberry-like" (or sometimes even fractal) rather than truly spherical shapes $[18,26]$. Indeed, TEM imaging of particles prepared at the $\mathrm{pH}$ and ionic strength used herein revealed a raspberry-like morphology, with clearly visible nanoscale subunits [26] (see Fig. 7). Thus, the radii of curvature governing the electrostatic repulsion between the particles may be closer to the 15-nm radius of the primary particles [26] than to the 80-nm radius of the secondary particles (see Table 1 and Fig. 8a). This view is supported by the nanometer-scale range of the repulsive potential shown in Fig. 6a, which reflects the short Debye 
screening length and makes it likely for only the subunits that are closest to the neighboring particle to contribute to the $\phi_{E}$. Repeating the calculations in Fig. 6 using this new radius of curvature yields much lower repulsive potentials (all below $7 k_{B} T$; see Supplementary Data, Fig. $\mathrm{S} 2 \mathrm{~b}-\mathrm{f})$ and theoretical collision rate constants that now exceed the experimental $k_{11}$-values (Supplementary Data, Fig. S3). When the $\mathrm{NaCl}$ concentration increases from 75 to $175 \mathrm{mM}$, these new theoretical collision rates increase from $7.2 \times 10^{-20}$ to $9.6 \times 10^{-20} \mathrm{~m}^{3} / \mathrm{s}$ when $A_{121}$ is estimated as $10^{-22} \mathrm{~J}$, and from $4.5 \times 10^{-19}$ to $8.4 \times 10^{-19} \mathrm{~m}^{3} / \mathrm{s}$ when $A_{121}$ is increased tenfold to $10^{-}$ ${ }^{21} \mathrm{~J}$. All of these values exceed the $4.6 \times 10^{-23}-1.5 \times 10^{-20} \mathrm{~m}^{3} / \mathrm{s}$ experimental $k_{11}$-values (see Table 1 and Fig. 6b) and, because aggregation events are expected to be less frequent than collisions, suggest that the raspberry-like particle shape might indeed (at least partly) account for the higher experimental aggregation rates.
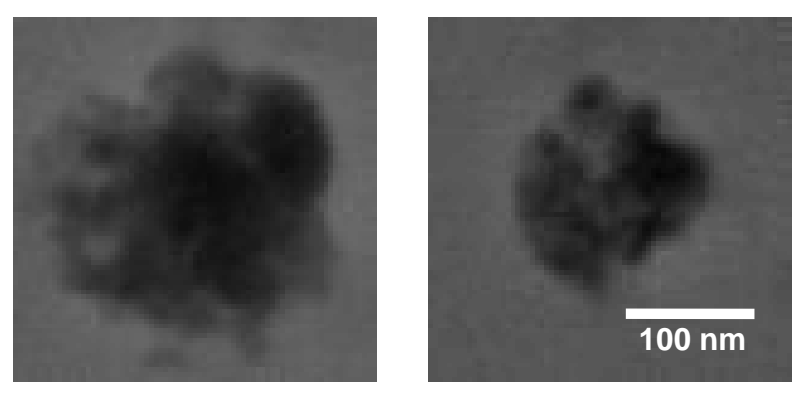

Fig. 7. TEM images of representative chitosan/TPP microgels prepared in $150 \mathrm{mM} \mathrm{NaCl}$ (parent chitosan and TPP solution $\mathrm{pH}=4.0$ ) from $90 \%$ DD chitosan. TPP:glucosamine ratio $=0.06: 1$ (reproduced from Ref. [26] with permission from the American Chemical Society). 

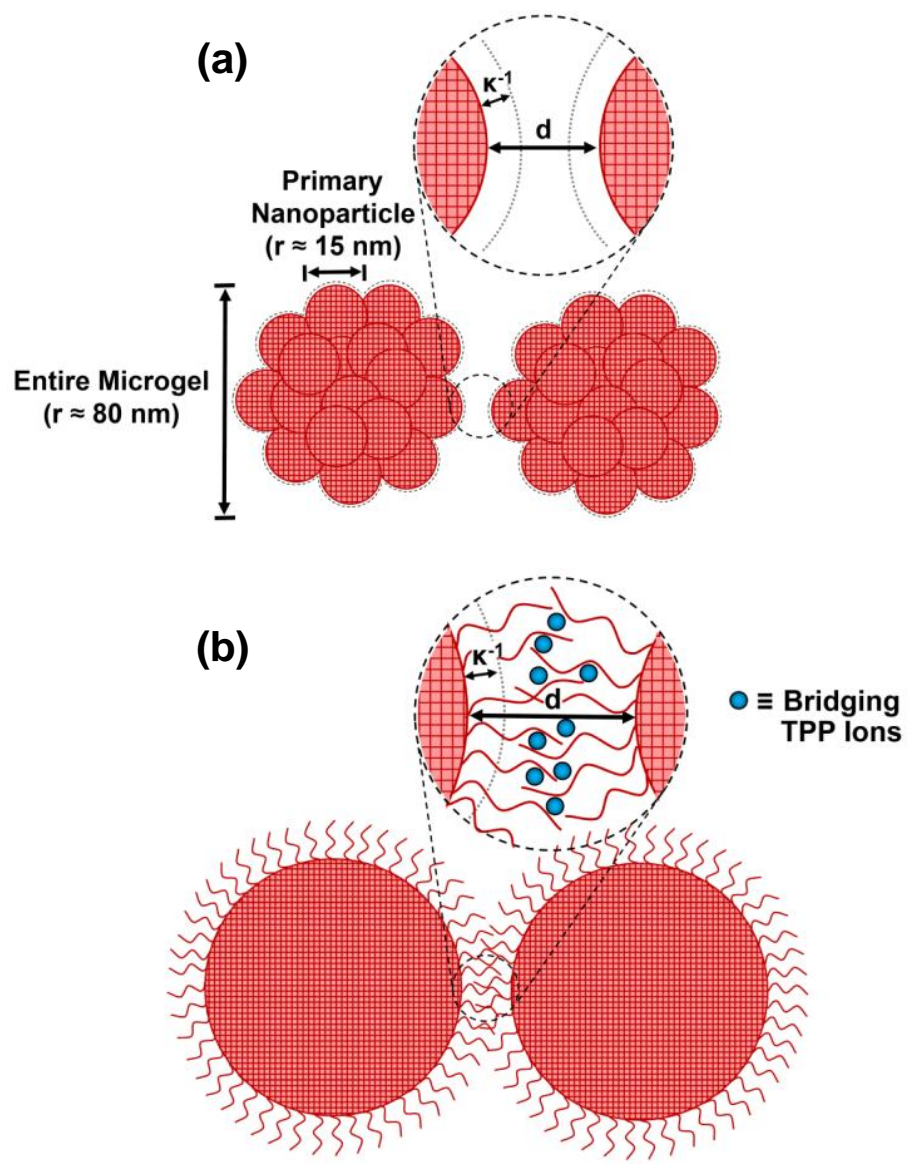

Fig. 8. The schematic diagrams of: (a) raspberry-like chotosan/TPP micro- and nanogel morphologies; and (b) chitosan chains extending from the particle surfaces beyond the Debye length $\left(\kappa^{-1}\right)$ and being bridged by TPP ions.

As a second explanation, we postulate that the faster experimental aggregation rates also may have reflected the bridging of the polymer segments extending from the particle surfaces (Fig. 8b). Instead of having a rigid and well-defined outer boundary that is modeled by the DLVO and Fuchs theories, polymeric microgels (albeit covalently crosslinked ones) have been reported to have dangling polymer chains that can extend out far beyond the polymer-rich core [58], and the same might exist for gel-like chitosan/TPP particles. The bare persistence length of chitosan is estimated at $6 \mathrm{~nm}[59,60]$, which is much greater than the roughly 1-nm Debye length in the aggregation experiments. Thus, the extended chitosan segments may easily extend beyond the double layer, especially at the $75-175 \mathrm{mM} \mathrm{NaCl}$ concentrations (where the double 
layer is highly compressed). These extended polyelectrolyte segments might therefore be bridged by TPP without the particles having to fully overcome the repulsive energy barrier (i.e., surfaceto-surface contact might not be required for bridging to occur). Accordingly, aggregation events might occur more frequently than the surface-to-surface collisions predicted by the Fuchs theory.

A related, third explanation is that this "soft" particle-solvent boundary may also have caused the $\psi_{0}$-values (which were used to estimate $\phi_{E}$ ) to be substantially lower than the measured $\zeta$-potentials. This effect may further limit the reliability of the DLVO-based $k_{11^{-}}$ predictions. Though this $\phi_{E}$ estimate could have potentially been improved using the Ohshima model [52, 53], reliable fitting of electrophoretic mobility data to this model required: (1) knowledge of the charged outer (brush) layer thickness; and (2) electrophoretic mobility measurements at much lower (ca. $1-10 \mathrm{mM}) \mathrm{NaCl}$ concentrations. Since structural information about the outer layer thickness was unavailable (and the impact of the 10 to 100-fold reduction in $\mathrm{NaCl}$ concentration on this structure was uncertain), this approach was not pursued in this initial aggregation kinetics study.

The fourth and final proposed explanation for why model $k_{11}$-values in Fig. 6b underpredict the aggregation rates is that collisions are more rapid due to charge heterogeneity on the chitosan/TPP particle surface. Surface charge heterogeneity (which is not accounted for by the DLVO theory) can generate additional attraction between particles and has been proposed as the reason for accelerated aggregation of polystyrene latex particles in the presence of tri- and tetravalent counterions (where aggregation rates were also faster than those predicted based on DLVO potentials) [61, 62]. Thus, it is possible that uneven TPP adsorption may lead to a heterogeneous chitosan/TPP particle charge and, just like in the case of the polystyrene latexes $[61,62]$, may cause a reduction in electrostatic repulsion. Though there is no direct evidence of 
surface charge heterogeneity-mediated attraction in chitosan/TPP dispersions, this possibility cannot be excluded.

3.6. Effects on Particle Polydispersity. Despite the above challenges in predicting $k_{11}$, the new insights on chitosan/TPP particle aggregation kinetics gained through this study can be used to develop better particle preparation procedures that optimize their size and polydispersity. During particle formation, where (like in the aggregation experiments performed here) there is initially free TPP present, both primary and higher-order chitosan/TPP particles are subject to TPPinduced aggregation. To ensure the formation of particles with uniform size distributions, both particle formation and aggregation should proceed at the same rate throughout the sample. A challenge in achieving this, however, is that chitosan/TPP particles often form faster than the time required to mix the parent chitosan and TPP solutions (which causes significant particle formation/aggregation to occur before TPP is distributed throughout the sample).

One way to overcome this problem is to slow the particle formation/aggregation process down, so that the particle aggregation during mixing is limited. We have recently shown that this effect can be achieved by preparing the chitosan/TPP particles in the presence of $150 \mathrm{mM} \mathrm{NaCl}$ (which drastically reduced particle polydispersity) [19]. The present findings, which show aggregation kinetics to also be slower at lower TPP concentrations, and lower $\mathrm{pH}$ and DD-values, suggest that similar effects may also be achieved by tuning other formulation parameters. To start exploring this, we have prepared particles at variable DD-values and matching chitosan and TPP concentrations (see Fig. 9). DLS analysis of these particles revealed their size distributions to become progressively more uniform at lower DD-values (where aggregation kinetics were slower), which supports the above hypothesis. 
(a)

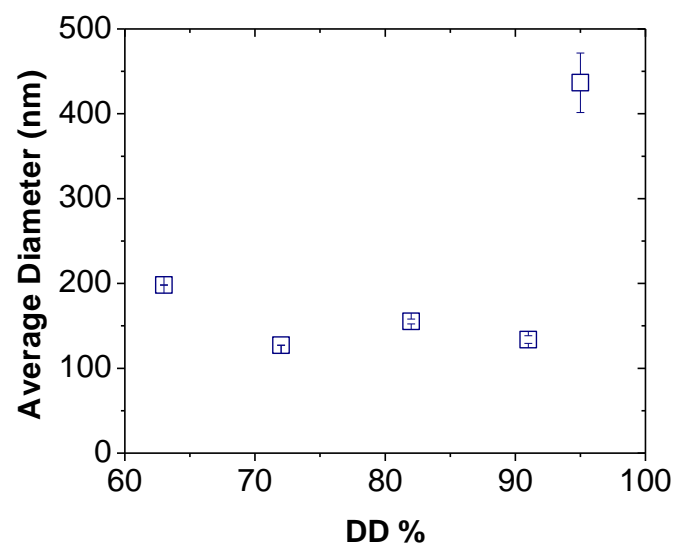

(b)

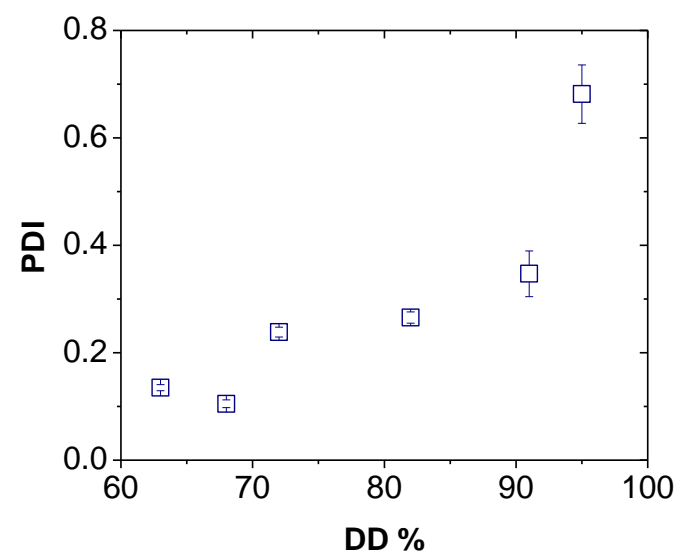

Fig. 8. DLS data showing estimates of the: (a) z-average hydrodynamic diameters and (b) PDIvalues of chitosan/TPP particles prepared from variably deacetylated chitosans at $\mathrm{pH} 4.0$ (with no added $\mathrm{NaCl}$ ) by adding $4 \mathrm{~mL}$ of $0.1 \mathrm{wt} \%$ TPP solution into $10 \mathrm{~mL}$ of $0.1 \mathrm{wt} \%$ chitosan. The error bars are standard deviations $(n=3)$.

In making the above adjustments to the DD-values (or other formulation parameters), however, several complications can occur. The first reflects the fact that conditions for slow aggregation coincide with those where chitosan/TPP binding is the weakest. These conditions can either destabilize chitosan/TPP particles (i.e., cause them to dissolve) or simply interfere with their formation. At low DD-values, for instance (e.g., at 63\% DD), the particles do not start forming until much higher TPP:glucosamine ratios than those required for their higher-DD counterparts [42]. Accordingly, since particles that are not fully-formed are more polydisperse $[19,26]$, lowering the DD to the point where particle formation is inhibited can lead to higher (rather than lower) apparent PDIs. Similar effects can occur in the limit of high $\mathrm{NaCl}$ concentrations, which can inhibit chitosan/TPP binding and (especially at lower DD-values [42]) prevent the particles from forming [19]. In such situations, where the addition of salt causes particles to be less-fully formed, the polydispersity in the presence of salt might be higher than in its absence. 
Another situation where $\mathrm{NaCl}$ addition fails to lower chitosan/TPP particle polydispersity is where very highly deacetylated $(\geq 95 \% \mathrm{DD})$ chitosan is used. Though adding $\mathrm{NaCl}$ still led to slower aggregation in the excess of TPP even when 95\% DD chitosan was used (Supplementary Data, Fig. S4), the addition of $\mathrm{NaCl}$ to these particles destabilized them under the TPP-limited conditions that are typically used in their preparation (i.e., below 0.2:1 TPP:glucosamine molar ratios, where without salt the particles remain stable). Instead of remaining stably dispersed at these TPP:glucosamine ratios (as when prepared under the salt-free condition), the particles prepared in $150 \mathrm{mM} \mathrm{NaCl}$ started noticeably aggregating over the timescales of minutes or hours, even when TPP was not in excess. Thus, when the chitosan DD-value was raised to $95 \%, \mathrm{NaCl}$ addition to the parent chitosan and TPP solutions no longer reliably reduced the particle PDI, since $\mathrm{NaCl}$ caused the particles to aggregate into polydisperse flocs (data not shown).

Remarkably, this salt effect at high DD-values under TPP-limited conditions was opposite to that seen when TPP was in excess (see Fig. S4). This might be because the weakened chitosan/TPP binding in the presence of $\mathrm{NaCl}$ [19] increased the free TPP concentration that remained unbound - i.e., it increased the chitosan/TPP dissociation constant. When TPP was added in excess (as done in the $k_{11}$-measurements), this likely had a negligible impact on the free TPP concentration, and thus did not affect the aggregation rates. Under TPP-limited conditions, however, there were only trace amounts of free TPP. Hence, the free TPP concentration likely increased much more appreciably due to the weakened chitosan/TPP binding in the presence of $\mathrm{NaCl}$. Because aggregation rates were acutely sensitive to the free TPP concentrations (see Fig. 3), this increase in free TPP might have diminished the extent to which $\mathrm{NaCl}$ addition inhibited particle bridging. Accordingly, under TPP-limited conditions the salt-induced inhibition of particle bridging became insufficient to outweigh the increased particle collision frequency 
caused by the electrostatic screening and, for particles prepared from 95\% DD chitosan (which, as suggested by Fig. 5b, aggregated appreciably even at low free TPP concentrations), $\mathrm{NaCl}$ accelerated particle aggregation and increased polydispersity.

These findings were consistent with those reported by other labs (where monovalent salt increased the PDI-values of particles prepared from 95 - 97.5\% DD chitosan) [18, 63], and likely reflected the stronger chitosan/TPP binding at higher DD-values [42]. When particles were formed from $91 \%$ (or lower) DD chitosan [19, 35], even if the increased frequency in chitosan/TPP particle collisions at elevated ionic strengths outweighed the NaCl-mediated reduction in bridging probability, bridging events at trace free TPP concentrations were very improbable. Thus, at least on the typical timescales of particle formation and characterization, the newly-formed particles did not aggregate into polydisperse flocs in the presence of $\mathrm{NaCl}$ unless TPP was in excess $[19,22]$. Conversely, when particles were prepared from $95-97.5 \%$ DD chitosan, the stronger chitosan/TPP binding evidently increased the bridging probability, and (when $\mathrm{NaCl}$ addition increased their collision frequency) caused polydisperse particle aggregates to form over experimental timescales. This DD-value effect explains the apparent discrepancies between reports that show monovalent salt to increase the PDI-values of chitosan/TPP particles (which used $95-97.5 \%$ DD chitosan) $[18,63]$ and reports that showed the monovalent salt to decrease the PDI and enhance the colloidal stability of chitosan/TPP particles (which used 83 90\% DD chitosan) $[19,35]$. In other words, at very high DD-values the trace amounts of TPP that remained available to bind to the particle surface at TPP:glucosamine ratios below 0.2:1 were sufficient to result in bridging flocculation, even when the chitosan/TPP binding was weakened by monovalent salt, and (unlike the chitosan/TPP particles with lower DD-values) caused the dispersions to be destabilized by $\mathrm{NaCl}$ addition. This effect, along with those of $\mathrm{NaCl}$, 
$\mathrm{pH}$ and DD on particle dissolution stability [42], must be carefully considered when tailoring chitosan/TPP micro- and nanogel formation/aggregation kinetics.

\section{Conclusions}

To quantify the aggregation events that determine chitosan/TPP micro- and nanogel size distributions [26, 27], chitosan/TPP microgel aggregation rate constants were measured for the first time. The aggregation rates increase sharply with the free TPP concentration and decrease sharply with the monovalent salt concentration. Likewise, the aggregation rates increase with the $\mathrm{pH}$ (at least while the $\mathrm{pH}$ remains far below the effective chitosan $\mathrm{p} K_{a}$ ) and with the chitosan degree of deacetylation (DD). These variations in aggregation rates apparently reflect the variations in the strength of TPP binding to the particle surface and, thus, its ability to bridge the particles together. Moreover, because these variations in aggregation rates were obtained using particles with the same size, they confirm that the monovalent salt-induced stabilization of chitosan/TPP dispersions is caused by weakened TPP binding [19], and not (as alternatively proposed) changes in chitosan/TPP particle size and compactness [35].

Interestingly, the measured aggregation rates are much faster than those predicted based on Derjaguin and Landau, Verwey and Overbeek (DLVO) interaction potentials. This might either reflect the presence of polymer segments extending from the particle surface, nonuniformities in particle shape (which is raspberry-like rather than spherical) and charge, or uncertainty in their overall surface potential estimate. These complicating factors, along with the observed disparity between experiment and theory, highlight the complexity in theoretically predicting the aggregation rate constants for ionically crosslinked micro- and nanogels. 
Also analyzed were the implications of these aggregation kinetics on the uniformity of chitosan/TPP micro- and nanogel size, which: (1) revealed how particle polydispersity can be diminished by lowering the chitosan DD; and (2) suggested that the opposing results on how chitosan/TPP particle polydispersity is affected by monovalent salt $[18,19]$ are likely a chitosan DD effect. Aside from tuning polydispersity, the improved understanding of the parameters governing chitosan/TPP particle aggregation may enable better strategies for tuning the average size of chitosan/TPP particles (and possibly other ionically crosslinked colloids [64]). Similarly, the aggregation rate constants obtained herein may ultimately enable predictive, theoretical (i.e., population balance [65]) modelling of chitosan/TPP micro- and nanogel formation, which could provide even better guidelines for tailoring their sizes to their various potential applications.

Acknowledgement. We are grateful to the National Science Foundation (CBET-1133795) for supporting this work and Dr. Michael Rubinstein (Univ. of North Carolina) for a helpful discussion.

\section{References:}

[1] Calvo P, Remunan-Lopez C, Vila-Jato JL, Alonso MJ. Chitosan and chitosan/ethylene oxidepropylene oxide block copolymer nanoparticles as novel carriers for proteins and vaccines. Pharm Res 1997;14:1431-6.

[2] Calvo P, Remunan-Lopez C, Vila-Jato JL, Alonso MJ. Novel hydrophilic chitosan-polyethylene oxide nanoparticles as protein carriers. J Appl Polym Sci 1997;63:125-32.

[3] Csaba N, Koping-Hoggard M, Alonso MJ. Ionically crosslinked chitosan/tripolyphosphate nanoparticles for oligonucleotide and plasmid DNA delivery. Int J Pharm 2009;382:205-14.

[4] De Campos AM, Sanchez A, Alonso MJ. Chitosan nanoparticles: A new vehicle for the improvement of the delivery of drugs to the ocular surface. Int J Pharm 2001;224:159-68.

[5] Gan Q, Wang T. Chitosan nanoparticle as protein delivery carrier-systematic examination of fabrication conditions for efficient loading in release. Colloids Surf B 2007;59:24-34.

[6] Garcia-Fuentes M, Alonso MJ. Chitosan-based drug nanocarriers: Where do we stand? J Controlled Release 2012;161:496-504.

[7] Ma Z, Yeoh HH, Lim LY. Formulation $\mathrm{pH}$ modulates the interaction of insulin with chitosan nanoparticles. J Pharm Sci 2002;91:1396-404. 
[8] Wang X, Chi N, Tang X. Preparation of estradiol chitosan nanoparticles for improving nasal absorption and brain targeting. Eur J Pharm Biopharm 2008;70:735-40.

[9] Colonna C, Conti B, Perugini P, Pavanetto F, Modena T. Chitosan glutamate nanoparticles for protein delivery: Development and effect on prolidase stability. J Microencapsulation 2007;24:553-64.

[10] Lehr CM, Bouwstra JA, Schacht EH, Junginger HE. In vitro evaluation of mucoadhesive properties of chitosan and some other natural polymers. Int J Pharm 1992;78:43-8.

[11] Vllasaliu D, Exposito-Harris R, Heras A, Casettari L, Garnett M, Illum L, et al. Tight junction modulation by chitosan nanoparticles: Comparison with chitosan solution. Int J Pharm 2010;400:183-93.

[12] de Campos AM, Diebold Y, Carvalho ELS, Sanchez A, Alonso MJ. Chitosan nanoparticles as new ocular drug delivery systems: in vitro stability, in vivo fate, and cellular toxicity. Pharm Res 2004;21:80310 .

[13] Fernandez-Urrusuno R, Calvo P, Remunan-Lopez C, Vila-Jato JL, Alonso MJ. Enhancement of nasal absorption of insulin using chitosan nanoparticles. Pharm Res 1999;16:1576-81.

[14] Hu B, Pan CL, Sun Y, Hou ZY, Ye H, Zeng XX. Optimization of fabrication parameters to produce chitosan-tripolyphosphate nanoparticles for delivery of tea catechins. J Agric Food Chem 2008;56:74518 .

[15] Katas H, Alpar HO. Development and characterisation of chitosan nanoparticles for siRNA delivery. J Controlled Release 2006;115:216-25.

[16] Fan W, Yan W, Xu ZS, Ni H. Formation mechanism of monodisperse, low molecular weight chitosan nanoparticles by ionic gelation technique. Colloids Surf B 2012;90:21-7.

[17] Gan Q, Wang T, Cochrane C, McCarron P. Modulation of surface charge, particle size and morphological properties of chitosn-TPP nanoparticles intended for gene delivery. Colloids Surf B 2005;44:65-73.

[18] Bugnicourt L, Alcouffe P, Ladaviere C. Elaboration of chitosan nanoparticles: Favorable impact of a mildthermal treatment to obtain finely divided, spherical, and colloidally stable objects. Colloids Surf A 2014;457:476-86.

[19] Huang Y, Lapitsky Y. Monovalent salt enhances colloidal stability during the formation of chitosan/tripolyphosphate microgels. Langmuir 2011;27:10392-9.

[20] Nasti A, Zaki NM, de Leonardis P, Ungphaiboon S, Sansongsak P, Rimoli MG, et al. Chitosan/TPP and chitosan/TPP-hyaluronic acid nanoparticles: Systematic optimisation of the preparative process and preliminary biological evaluation. Pharm Res 2009;26:1918-30.

[21] Zhang H, Oh M, Allen C, Kumacheva E. Monodisperse chitosan nanoparticles for mucosal drug delivery. Biomacromolecules 2004;5:2461-8.

[22] Jonassen H, Kjoniksen AL, Hiorth M. Effects of ionic strength on the size and compactness of chitosan nanoparticles. Colloid Polym Sci 2012;290:919-29.

[23] Grenha A, Seijo B, Remunan-Lopez C. Microencapsulated chitosan nanoparticles for lung protein delivery. Eur J Pharm Sci 2005;25:427-37.

[24] Fernandez-Urrusuno R, Romani D, Calvo P, Vila-Jato JL, Alonso MJ. Development of a freeze-dried formulation of insulin-loaded chitosan nanoparticles intended for nasal administration STP Pharm Sci 1999;9:429-36.

[25] Tsai ML, Bai SW, Chen RH. Cavitation effects versus stretch effects resulted in different size and polydispersity of ionotropic gelation chitosan-sodium tripolyphosphate nanoparticle. Carbohyd Polym 2008;71:448-57.

[26] Huang Y, Lapitsky Y. Salt-assisted mechanistic analysis of chitosan/tripolyphosphate micro- and nanogel formation. Biomacromolecules 2012;13:3868-76. 
[27] Lapitsky Y. Ionically crosslinked polyelectrolyte nanocarriers: Recent advances and open problems. Curr Opinion Colloid Interface Sci 2014;19:122-30.

[28] Hiemenz PC, Rajagoplan R. Principles of Colloid and Surface Chemistry. New York: Marcel Dekker, Inc.; 1997.

[29] Wu JZ, Huang G, Hu ZB. Interparticle potential and the phase behavior of temperature-sensitive microgel dispersions. Macromolecules 2003;36:440-8.

[30] Saunders BR, Vincent B. Microgel particles as model colloids: theory, properties and applications. Adv Colloid Interface Sci 1999;80:1-25.

[31] Israelachvili J, Wennerström H. Role of hydration and water structure in biological and colloidal interactions. Nature 1996;379:219-25.

[32] Pelton RH, Chibante P. Preparation of aqueous latices with N-isopropylacrylamide. Colloids Surf 1986;20:247-56.

[33] Wu JZ, Huang G, Hu ZB. Phase behavior of thermally responsive microgel colloids Phys Rev Lett 2003;90:048304.

[34] Li J, Huang Q. Rheological properties of chitosan-tripolyphosphate complexes: From suspensions to microgels Carbohyd Polym 2012;87:1670-7.

[35] Jonassen H, Kjoniksen AL, Hiorth M. Stability of chitosan nanoparticles cross-linked with tripolyphosphate. Biomacromolecules 2012;13:3747-56.

[36] Behrens SH, Christl DI, Emmerzael R, Schurtenberger P, Borkovec M. Charging and aggregation properties of carboxyl latex particles: Experiments versus DLVO theory. Langmuir 2000;16:2566-75.

[37] Holthoff H, Egelhaaf SU, Borkovec M, Schurtenberger P, Sticher H. Coagulation rate measurements of colloidal particles by simultaneous static and dynamic light scattering. Langmuir 1996;12:5541-9.

[38] Van Zanten JH, Elimelech M. Determination of absolute coagulation rate constants by multiangle light scattering. J Colloid Interface Sci 1992;154:1-7.

[39] Garcia-Salinas M, Romero-Cano M, De Las Nieves F. Colloidal stability of a temperature-sensitive poly (N-isopropylacrylamide/2-acrylamido-2-methylpropanesulphonic acid) microgel. J Colloid Interface Sci 2002;248:54-61.

[40] Deryagin B, Landau L. Theory of the stability of strongly charged lyophobic sols and of the adhesion of strongly charged particles in solutions of electrolytes. Acta Physicochim URSS 1941;14:633-62.

[41] Verwey EJW, Overbeek JTG. Theory of the stability of lyophobic colloids. J Colloid Sci 1955; 10:224-5.

[42] Huang Y, Cai Y, Lapitsky Y. Factors affecting the stability of chitosan/tripolyphosphate micro- and nanogels: Resolving the opposing findings. J Mater Chem B 2015;3:5957-70.

[43] Lapitsky Y, Zahir T, Shoichet MS. Modular biodegradable biomaterials from surfactant and polyelectrolyte mixtures. Biomacromolecules 2008;9:166-74.

[44] Fuchs N. Uber die stabilitat und aufladung der aerosole. Z Physik 1934;89:736-43.

[45] Holthoff H, Schmitt A, Fernandez-Barbero A, Borkovec M, Cabrerizo-Vilchez MA, Schurtenberger $\mathrm{P}$, et al. Measurement of absolute coagulation rate constants for colloidal particles: Comparison of single and multiparticle light scattering techniques. J Colloid Interface Sci 1997;192:463-70.

[46] Robert J. Hunter, R. H. Ottewill, R. L. Rowell. Zeta Potential in Colloid Science. New York: Elsevier Ltd.; 1981.

[47] Cai Y, Lapitsky Y. Formation and dissolution of chitosan/pyrophosphate nanoparticles: Is the ionic crosslinking of chitosan reversible? Colloids Surf B 2014;115:100-8. 
[48] Pillai CKS, Paul W, Sharma CP. Chitosan and chitosan polymers: Chemistry, solubility and fiber formation. Prog Polym Sci 2009;34:641-78.

[49] Sorlier P, Denuziere A, Viton C, Domard A. Relation between the degree of acetylation and the electrostatic properties of chitin and chitosan. Biomacromolecules 2001;2:765-72.

[50] Laus R, Costa TG, Szpoganicz B, Favere VT. Adsorption and desorption of $\mathrm{Cu}$ (II), $\mathrm{Cd}$ (II) and $\mathrm{Pb}$ (II) ions using chitosan crosslinked with epichlorohydrin-triphosphate as the adsorbent. J Hazard Mater 2010;183:233-41.

[51] Lawrence PG, Lapitsky Y. Ionically crosslinked poly(allylamine) as a stimulus-responsive underwater adhesive: Ionic strength and pH effects. Langmuir 2015;31:1564-74.

[52] Ohshima H. Electrokinetics of soft particles. Colloid Polym Sci 2007;285:1411-21.

[53] Ohshima H, Kondo T. Approximate analytic expression for the electrophoretic mobility of colloidal particles with surface-charge layers. J Colloid Interface Sci 1989;130:281-2.

[54] Russel WB, Saville DA, Schowalter WR. Colloidal Dispersions. Cambridge: Cambridge University Press; 1989.

[55] Honig EP, Roebersen GJ, Wiersema PH. Effect of hydrodynamic interaction on the coagulation rate of hydrophobic colloids. J Colloid Interface Sci 1971;36:97-109.

[56] Israelachvili J. Intermolecular \& Surface Forces. 2nd ed. London: Academic Press; 1992.

[57] Holmberg M, Berg J, Stemme S, Oedberg L, Rasmusson J, Claesson P. Surface force studies of Langmuir-Blodgett cellulose films. J Colloid Interface Sci 1997;186:369-81.

[58] Eckert T, Richtering W. Thermodynamic and hydrodynamic interaction in concentrated microgel suspensions: Hard or soft sphere behavior? J Chem Phys 2008;129:124902/1-/6.

[59] Kayitmazer AB, Shaw D, Dubin PL. Role of Polyelectrolyte Persistence Length in the Binding of Oppositely Charged Micelles, Dendrimers, and Protein to Chitosan and Poly(dimethyldiallyammonium chloride). Macromolecules 2005;38:5198-204.

[60] Berth G, Colfen H, Dautzenberg H. Physicochemical and chemical characterisation of chitosan in dilute aqueous solution. In: Borchard E, Straatmann A, editors. Analytical Ultracentrifugation Vi. Berlin: Springer-Verlag Berlin; 2002. p. 50-7.

[61] Sinha P, Szilagyi I, Ruiz-Cabello FJM, Maroni P, Borkovec M. Attractive forces between charged colloidal particles induced by multivalent ions revealed by confronting aggregation and direct force measurements. J Phys Chem Lett 2013;4:648-52.

[62] Szilagyi I, Polomska A, Citherlet D, Sadeghpour A, Borkovec M. Charging and aggregation of negatively charged colloidal latex particles in the presence of multivalent oligoamine cations. J Colloid Interface Sci 2013;392:34-41.

[63] Wu Y, Yang W, Wang C, Hu J, Fu S. Chitosan nanoparticles as a novel delivery system for ammonium glycyrrhizinate. Int J Pharm 2005;295:235-45.

[64] Pistone S, Qoragllu D, Smistad G, Hiorth M. Formulation and preparation of stable cross-linked alginate-zinc nanoparticles in the presence of a monovalent salt. Soft Matter 2015;11:5765-74.

[65] Eitzlmayr A, Petschacher C, Radl S, Suzzi D, Zimmer A, Khinast JG. Modeling and simulation of polyacrylic acid/protamine nanoparticle precipitation. Soft Matter 2011;7:9484-97. 